\title{
RhPDGF - BASIC CHARACTERISTICS AND POTENTIAL APPLICATION IN THE ORAL SURGERY - AN OVERVIEW
}

\author{
Z. Mihaylova, R. Ugrinov, E. Aleksiev, P. Stanimirov \\ Department of Dental, Oral and Maxillofacial Surgery, Faculty of Dental Medicine, \\ Medical University - Sofia, Bulgaria
}

\begin{abstract}
Growth factors (GFs) are bioactive molecules participating in organ development, tissue regeneration and repair. They are protein molecules with a relatively low molecular weight and are released by activated platelets. Platelet-derived growth factor (PDGF) is one of the GFs of highest amount in human platelets. It is known to stimulate cell proliferation and extracellular matrix synthesis, as well as angiogenesis in healthy tissues and neoplasms. However, most of the studies in the literature demonstrate the influence of PDGF on tissue regeneration without revealing its intimate mechanisms of action on different cell types. In the current review we emphasis on the effects of PDGF in order to stimulate various biological processes in wide number of pre-clinical and clinical studies.
\end{abstract}

Key words: growth factors, platelet-derived growth factor, PDGF, oral surgery

Corresponding author: Zornitsa Mihaylova, DDS, PhD, e-mail:dr.z.mihaylova@gmail.com

\section{GROWTH FACTORS}

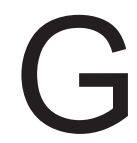
rowth factors (GFs) are active substances that participate in all biological processes. They are isolated from various cell types, but are found to be of highest amount in the platelets. Some well-known GFs, able to stimulate variety of cell functions are platelet-derived growth factor (PDGF), vascular endothelial growth factor (VEGF), transforming growth factor beta (TGF- $\beta$ ), fibroblast growth factor (FGF), epidermal growth factor (EGF), etc.

GFs are small proteins that bind to cell surface receptors [1]. Thus, a specific cellular activity is maintained. Up to date, scientists have revealed the potential of the GFs to stimulate and accelerate tissue repair and regeneration [2, 3]. We have also investigated the effects of some of the most abundant GFs found in human platelets on different cell types, including dental stem cells, oral mucosa keratinocytes, fibroblasts, etc. $[4,5]$.

The function of the GFs is hardly dependent on the following factors: their concentration and the half-life of each GF. Multiple active molecules from the local environment are also capable of modifying the biological activity of the GFs [6]. The effects of the GFs are also known to be influenced by the specific phenotype of the target cells [7].

Currently GFs are highly researched substances in the field of medicine and dentistry due to their key function in tissue regeneration. Probably the main purpose of all preclinical and clinical studies conducted is to reveal the optimal dose of the applied GF, as well as to introduce proper techniques for slow molecular release in the living tissues. Therefore, various biomaterials are used as scaffolds in order to reveal the most favorable variant for tissue regenera- 
tion [8]. These scaffolds (also called matrices) when loaded with active molecules (i.e. GFs) may serve as a depot for their slow release, as well as to maintain high local concentration for sufficient number of hours or days. Some of the most commonly used scaffolds are alginate, gelatin, autologous fibrin glue, polyglycolic acid, etc. [9]. There are specific criteria when choosing the most appropriate scaffold for particular in vitro and in vivo studies, as follows: to be easy to apply, to be able to provide long-term stable scaffold for cell colonization, to be inexpensive, etc [9].

Recombinant GFs are used in many fields of dentistry and medicine, including oral and maxillofacial surgery, dental implantology, periodontal surgery, orthopedics, regenerative medicine, treatment of nonhealing ulcers (diabetic ulcers), etc. [8, 10]. The effects of GFs as single molecules or in combination with other substances have been reported in wide number of clinical trials. However, more detailed in vitro experiments with recombinant GFs should be conducted in order to reveal their intimate mechanisms of action. Thus, more predictable clinical outcomes of their local application are expected.

\section{PLATELET-DERIVED GROWTH FACTOR - PDGF}

PDGF is one of the most abundant GFs found in human platelets. Other cell types when stimulated, including non-differentiated cells, smooth muscle cells, endothelial cells, macrophages are able to produce and to release it in the local environment [11]. PDGF is situated in the platelets alpha-granules [12]. As the rest of the GFs it is released after degranulation of the activated platelets. They are found of high amount in the blood clot after tissue injury [13]. PDGF, in particular, is a GF with relatively short half-life and once released from the activated platelets, it's molecule remains active for the next 30 minutes [14].

PDGF is found as a homo- or heterodimeric molecule [15]. PDGF may consist of the following polypeptide chains: $A, B, C$ or $D$ linked together with a disulfide bridge [16]. It's isomeric forms are PDGF-AA, -BB, $-A B,-C C,-D D$. The PDGF isomers may possess different biologic activities.

GF's secretion from particular cells might be modified when stimulated with other substances. We have observed increased production of PDGF (PDGF-AA in particular) in cells when stimulated with a combination of other specific GFs (data not shown). However, our research on this topic is still at a very early stage. Our aim is to reveal as many details as possible about the effects of several GFs on dental stem cells, oral keratinocytes and fibroblasts.
PDGF is able to interact with two distinct types of highly-specific cellular receptors. These receptors are known as alpha and beta-receptors and are found on the cell surface [17]. When PDGF bind to its receptors, specific signal pathways are triggered within the target cell, as multiple downstream substrates and cell regulatory proteins are activated. Thus, various processes like cell differentiation, proliferation and migration are established [18]. The effects of PDGF are dependent on the biology of the target cell, as each cell type may respond differently.

PDGF is among the active molecules that regulate various cell processes. It plays crucial role in the soft tissue and bone healing and regeneration; PDGF is a major mitogen for osteoblasts, undifferentiated cells, fibroblasts, etc.; PDGF stimulates migration of inflammatory cells towards the site of tissue damage; one of the main function of PDGF is stimulation of angiogenesis $[19,20]$. There are reports revealing the ability of PDGF to enhance the tissue healing via initiation and maintenance of collagen synthesis in cells [21], thus contributing to high mechanical resistance of tissues at the side of injury. We have also found that PDGF contributes to the collagen chains maturation in non-differentiated stem cells of human periodontal ligament [4].

PDGF participates in the development of neoplasms. It stimulates tumor neo-angiogenesis and increases the secretion of specific substances from tumor cells, responsible for various tissue alterations [22]. Therefore, multiple techniques based on the PDGF's activity suppression have been suggested, in order to prevent tumor growth and the development of metastatic disease.

\section{RECOMBINANT PDGF (RHPDGF)}

No data has been found yet in the literature regarding the application of human blood-isolated single GFs in vitro and in vivo. Multiple preclinical and clinical studies have been conducted to reveal the activity and the mechanism of action of the GFs. For this purpose recombinant molecules are used. Recombinant GFs are concentrated and purified to a high degree substances created for experimental and clinical application [23]. Preclinical and clinical trials demonstrating their effects on healing processes are performed [24, $25,26]$. Relatively new group of autologous blood derived products applied in the medical field are the platelet concentrates - platelet rich plasma, platelet rich fibrin and their analogues. In our opinion these products may serve as sources for GFs fractions isolation and further application for research and therapeutic purposes. However, currently we cannot present sufficient research data in this field. 
Limited number of recombinant growth factors (rhGFs) has been introduced as routine therapeutic agents in the medical practice nowadays. To the best of our knowledge, rhPDGF is the first recombinant GF allowed for clinical use by the American Food and Drug Administration (FDA). It has been first applied in the management of nonhealing diabetic ulcers on extremities [27]. Its high efficacy rate and no reports in the literature of local complications following its application have been documented [27].

Disadvantage of PDGF administration in the clinic refers to its short half-life. Increased concentration of the exogenous GF applied in tissues poses a risk of undesirable side effects [28], eventhough the clinical reports about the development of neoplasms following rhGF's application are limited. The problem with PDGF's short half-life has led to the development of biomaterials capable of forming a depot for a slow local release of the GF [29].

\section{EFFECT OF PDGF ON CELL FUNCTION}

The effects of PDGF on different cell types in vitro have been reported by many authors. Experiments predominantly reveal the mitogenic activity of the GF, as there are many studies showing positive effects of PDGF on cell proliferation. However, PDGF is also known to stimulate specific genetic expression, cell migration and extracellular matrix synthesis in fibroblasts, monocytes, macrophages and many other cell types $[13,19]$.

Boyan et al. [30] investigated the effect of three isomeric forms of PDGF (PDGF-AA, -BB and -AB) on cell proliferation rate and cell migration in human periodontal ligament cells. The cell culture used in the experiment is heterogeneous. The authors conclude that PDGF-BB exhibit significantly higher mitogenic properties when compared to the other molecules of the PDGF family [30]. The results reveal that different isoforms of a particular GF may have distinct properties and the most appropriate molecule should be chosen for particular purposes.

PDGF-AB has been reported to stimulate cell proliferation and migration, as well as the collagen synthesis of periodontal ligament cells in animal studies [31]. The GF's activity is highly dependent on the concentration applied. Our studies with rhPDGF-BB also reveal that it modifies various cell processes in human periodontal ligament stem cells in a concentration-dependent manner [4].
The effect of PDGF-BB on mesenchymal stem cells has been investigated in details in another in vitro study [32]. The authors reveal that optimal PDGF's activity on cell proliferation is established at a higher concentrations, whereas collagen production is more significant at a low concentration. Again the dose dependent efficacy of the GF on the cell biology is highlighted.

The effect of PDGF-BB on the activity of alkaline phosphatase (ALP) (early marker for osteogenic differentiation) has been investigated [33]. The results show that PDGF-BB increases the production of proteins from cells and inhibits ALP activity.

The effect of PDGF on osteogenic differentiation of undifferentiated cell lineages remains controversial. According to relatively recent reports, PDGF does not stimulate differentiation of human mesenchymal stem cells towards osteoblasts [34]. Strayhorn et al. [35], demonstrate the ability of PDGF to potentiate osteoblastic proliferation in vitro in a concentration-dependent manner. In the authors' opinion, PDGF does not enhance osteogenic cell differentiation, as its application blocks the expression of markers strictly related to osteogenic cell differentiation.

The proper tissue regeneration might be maintained experimentally by the action of multiple substances. Some substances participate synergistically and some of them are antagonists, therefore particular effects in cells and tissues are established. It has been reported that PDGF when added to cell culture media in combination with 2 or more GFs acting as its synergists, significantly enhances cell proliferation and adhesion when compared to PDGF applied alone [36].

\section{APPLICATION OF RHPDGF IN VIVO}

Recombinant GFs, usually in a combination with various tissue grafts are introduced in the dental clinical practice. Their effects following local application have been investigated in wide number of studies $[24,37]$. Main issues regarding the clinical use of the GFs again are their short half-life and rapidly decreased local concentration soon after their application [38]. On the other hand, the presence of other molecules that may reduce their activity should be considered. However, rhPDGF is a promising active molecule for tissue regeneration in the clinical practice, as it's role in various cell processes is well- 
known, i.e. cell proliferation, collagen chains maturation, etc. PDGF also stimulates angiogenesis and recruitment of cells from the surrounding healthy tissues [20]. The main purpose is to introduce rhPDGF into the field of the oral surgery for management of bone resorption around natural teeth and dental implants, socket preservation, guided bone regeneration, management of jaw-bone cavities after cystectomy, etc. PDGF is not applied locally as a sole molecule, but in combination with auto-, allo-, xenograft or synthetic material [39]. The grafts serve as a scaffold when additional mechanical support is needed at the side of the tissue repair and regeneration. On the other hand, the scaffold is colonized by high number fibroblasts and stem cells, as well as with sufficient amount of highly differentiated cells which is crucial for the ongoing regeneration at the site of tissue injury. Various grafts also maintain higher local concentration of the GF.

Limited number of clinical studies has been conducted using rhPDGF when compared to the reported in vitro experiments. rhPDGF at different concentrations in a combination with beta-tricalcium phosphate have been applied in the management of vertical bone defects [40]. The analysis of the clinical outcomes has been made 3 to 6 months postoperatively. The results reveal that only lower PDGF concentrations show promising effect on bone regeneration. Further experiments are needed to find out the optimal GF's concentration in each particular clinical experiment, which is of highly importance for the following tissue regeneration and rehabilitation.

Nevins et al. [41] administer PDGF with allogeneic lyophilised bone graft in the management of teeth with advanced bone resorption and high degree mobility. In the following 6 months, no signs of soft tissue inflammation were identified. Newly formed bone around the teeth was observed radiographically. Complete regeneration in both Class II furcations and interproximal intrabony defects managed was documented.

Chang et al. [42] revealed that PDGF-B gene therapy accelerates osseointegration of oral implants placed at extraction socket defects in animal models. Another study [43] demonstrates that the application of rhPDGF in combination with beta-tricalcium phosphate enhances the bone regeneration in critical size peri-implant bone defects. The defects showed the highest rate of bone formation 2 to 4 weeks postoperatively.
Kaigler et al. [44] in a literature review postulated that the current available evidence recommend the use of rhPDGF-enhanced matrices to promote periodontal and peri-implant bone regeneration.

PDGF can be applied locally at the side of the osteotomy prior immediate implant placemen in order to accelerate the osseointegration of the dental implants. Reports reveal complete osteointegration of the implants at the sides where PDGF-BB is applied together with alo- or xenograft [45].

Base on the documented clinical studies found, rhPDGF is a promising therapeutic agent in the field of the oral surgery in the development of innovative methods for the management of periodontal and periimplant defects, guided bone regeneration and osseointegration of dental implants.

\section{CONCLUSION}

Numerous approved and well-known oral surgical procedures, including distraction osteogenesis, guided bone regeneration, guided soft tissue regeneration, and autogeneous bone grafting, provide dentists with various approaches to the management of alveolar bone atrophy, periodontal defects compromised dental implant treatment, etc. Most of these treatment modalities show desirable clinical outcomes. However, multiple studies demonstrate limited success when routine approaches are used. Recently, in the field of regenerative medicine, innovative treatment methods are suggested. The combination of recombinantly produced growth factors and different scaffolds is a promising approach for the management of oral and dental pathology. Further research is needed prior routine application of these methods in order to achieve predictable clinical results.

Disclosure summary: The authors have nothing to disclose.

\section{REFERENCES:}

1. Sporn M, Roberts A. Peptide growth factors and their receptors I/II. Springer-Verlag Berlin Haidelberg 2012. ISBN9783642492952.

2. da Silva Meirelles L, Chagastelles PC, Nardi NB. Mesenchymal stem cells reside in virtually all post-natal organs and tissues. J Cell Sci 2006; 119: 2204-2213. 
3. Sanchez AR, Sheridan PJ, Kupp LI. Is platelet-rich plasma the perfect enhancement factor? A current review. Int J Oral Maxillofac Implants 2003; 18: 93-103.

4. Mihaylova Z, Tsikandelova R, Sanimirov P, et al. Role of PDGF-BB in proliferation, differentiation and maintaining stem cell properties of PDL cells in vitro. Arch Oral Biol 2018; 85, 1-9.

5. Tsikandelova, R, Mladenov, P, Planchon, S, et al. Proteome response of dental pulp cells to exogenous FGF8. J Proteom 2018; 183, 14-24.

6. Lesley J, Hascall VC, Tammi M, Hyman R. Hyaluronan binding by cell surface CD44. J Biol Chem 2000; 275: 26967-26975.

7. Chen FM, Shelton RM, Jin Y, Chapple IL. Localized delivery of growth factors for periodontal tissue regeneration: role, strategies, and perspectives. Med Res Rev 2009; 29: $472-$ 513.

8. Vasita R, Katti DS. Growth factor-delivery systems for tissue engineering: a materials perspective. Expert Rev Med Devices 2006; 3: 29-47.

9. Balasubramanian V, Onaca O, Enea R et al. Protein delivery: from conventional drug delivery carriers to polymericnano- reactors. Expert Opin Drug Deliv 2010; 7: 63-78.

10. Uebersax L, Merkle HP, Meinel L. Biopolymer-based growth factor delivery for tissue repair: from natural concepts to engineered systems. Tissue Eng Part B Rev 2009; 15: 263-289.

11. Kumar V. Robbins and Coltran pathologic basis of disease. 2010; China: Elsevier p.88-89. ISBN 978-1-4160-3121-5.

12. Michelson A. Platelets 3rd ed. St. Louis: Mosbi - Elsevier 2013 ISBN: 978-0-12-387837-3

13. Pierce GF, Mustoe TA, Altrock BW et al. Role of platelet-derived growth factor in wound healing. J Cell Biochem. 1991; 45: 319-326.

14. Lynch SE, Castilla GRD, Williams RC et al. The Effects of Short-Term Application of a Combination of Platelet-Derived and Insulin-Like Growth Factors on Periodontal Wound Healing. J Periodontol 1991; 62: 458-467.

15. Andrae J, Gallini R, Betsholtz C. Role of platelet-derived growth factors in physiology and medicine. Genes Dev 2008; 22: $1276-1312$.

16. Hart CE, Forstrom JW, Kelly JD et al. Two classes of PDGF receptor recognize different isoforms of PDGF. Science. 1988; 240: 1529-1531.

17. Gebhard C, Akhmedov A, Mocharla P et al. PDGF-CC induces tissue factor expression: role of PDGF receptor alpha/ beta. Basic Res Cardiol 2010; 105: 349-356.

18. Lemmon MA, Schlessinger J. Cell signaling by receptor tyrosine kinases. Cell 2010; 141: 1117-1134.

19. Alvarez RH, Kantarjian HM, Cortes JE. Biology of plateletderived growth factor and its involvement in disease. Mayo Clin Proc 2006; 81: 1241-1257.

20. Graham S, Leonidou A, Lester $M$ et al. Investigating the role of PDGF as a potential drug therapy in bone formation and fracture healing. Expert Opin Investig Drugs. 2009; 18: 1633-1654.

21. Taba M Jr, Jin Q, Sugai JV, Giannobile WV. Current concepts in periodontal bioengineering. Orthod Craniofac Res 2005; 8 : 292-302.

22. Cao Y. Multifarious functions of PDGFs and PDGFRs in tumor growth and metastasis. Trends Mol Med 2013; 19: 460-473.
23. Nevins M, Hanratty J, Lynch SE. Clinical results using recombinant human platelet-derived growth factor and mineralized freeze-dried bone allograft in periodontal defects. Int J Periodontics Restorative Dent 2007; 27: 421-427.

24. Camelo M, Nevins ML, Schenk RK, LynchSE, Nevins M. Periodontal regeneration in human Class II furcations using purified recombinant human platelet derived growth factor-BB (rhPDGF-BB) with bone allograft. Int J Periodontics Restorative Dent 2003; 23: 213-25.

25. Chowdhary KY, George JP, Gowda P, Rao JA. Human periodontal ligament fibroblast response to rhPDGF-BB application on periodontally diseased root surfaces-in vitro. Growth Factors 2013; 31: 130-138

26. Nevins $M$, Camelo $M$, Nevins $M L$ et al. Periodontal regeneration in humans using recombinant human platelet-derived growth factor-BB (rhPDGF-BB) and allogenic bone. J Periodontol 2003; 74: 1282-1292.

27. Wieman TJ, Smiell JM, Su Y. Efficacy and safety of a topical gel formulation of recombinant human platelet-derived growth factor- BB (becaplermin) in patients with chronic neuropathic diabetic ulcers. A phase III randomized placebo-controlled double-blind study. Diabetes Care 1998; 21: 822-827.

28. Mehrara BJ, Saadeh PB, Steinbrech DS et al. Adenovirusmediated gene therapy of osteoblasts in vitro and in vivo. $\mathrm{J}$ Bone Miner Res 1999; 14: 1290-1301.

29. Roy K, Mao HQ, Huang SK, Leong KW. Oral gene delivery with chitosan-DNA nanoparticles generates immunologic protection in a murine model of peanut allergy. Nat Med 1999; 5: 387-391.

30. Boyan LA, Bhargava G, Nishimura F et al. Mitogenic and chemotactic responses of human periodontal ligament cells to the different isoforms of plateletderived growth factor. J Dent Res 1994; 73: 1593-1600.

31. Matsuda N, Lin WL, Kumar NM et al. Mitogenic, chemotactic, and synthetic responses of rat periodontal ligament fibroblastic cells to polypeptide growth factors in vitro. J Periodontol 1992; 63: 515-525.

32. Ojima Y, Mizuno M, Kuboki Y, Komori T. In vitro effect of platelet-derived growth factor-BB on collagen synthesis and proliferation of human periodontal ligament cells. Oral Dis 2003; 9: 144-151.

33. Zaman $\mathrm{KU}$, Sugaya $\mathrm{T}$, Kato $\mathrm{H}$. Effect of recombinant human platelet-derived growth factor-BB and bone morphogenetic protein-2 application to demineralized dentin on early periodontal ligament cell response. J Periodontal Res 1999; 34: 244-250.

34. Kratchmarova I, Blagoev B, Haack-Sorensen M, Kassem $M$, Mann M. Mechanism of divergent growth factor effects in mesenchymal stem cell differentiation. Science. 2005; 308: 1472-1477.

35. Strayhorn CL, Garrett JS, Dunn RL et al. Growth factors regulate expression of osteoblastassociatedgenes. J Periodontol 1999; 70: 1345-1354.

36. Sant'Ana AC, Marques MM, Barroso TE et al. Effects of TGFbeta 1, PDGF-BB and IGF-1 on the rate of proliferation and adhesion of a periodontal ligament cell lineage in vitro. J periodontol 2007; 78: 2007-2017. 
37. Chen FM, Jin Y. Periodontal tissue engineering and regeneration: current approaches and expanding opportunities. Tissue Eng Part B Rev 2010; 16: 219-255.

38. Andreadis ST, Geer DJ. Biomimetic approaches to protein and gene delivery for tissue regeneration. Trends Biotechnol 2006; 24: 331-337.

39. Stephan EB, Renjen R, Lynch SE, Dziak R. Platelet-derived growth factor enhancement of a mineral-collagen bone substitute. J Periodontol 2000; 71: 1887-1892.

40. Nevins M, Giannobile WV, McGuire MK et al. Platelet-derived growth factor stimulates bone fill and rate of attachment level gain: results of a large multicenter randomized controlled trial. J Periodontol 2005; 76: 2205-2215.

41. Nevins $M$, Camelo $M$, Nevins $M L$, et al. Periodontal regeneration in humans using recombinant human platelet-derived growth factor-BB (rhPDGF-BB) and allogenic bone. J Periodontol 2003; 74(9), 1282-1292.
42. Chang PC, Seol YJ, Cirelli JA, et al. PDGF-B gene therapy accelerates bone engineering and oral implant osseointegration. Gene Ther 2010; 17(1), 95-104.

43. Choo T, Marino V, Bartold PM. Effect of PDGF-BB and betatricalcium phosphate ( $\beta$-TCP) on bone formation around dental implants: a pilot study in sheep. Clin Oral Implants Res 2013; 24(2), 158-166.

44. Kaigler, D, Avila, G, Wisner-Lynch, L, et al. Platelet-derived growth factor applications in periodontal and peri-implant bone regeneration. Exp Opin Biological Ther 2011; 11(3), 375-385.

45. McAllister BS, Haghighat K, Prasad HS, Rohrer MD. Histologic evaluation of recombinant human platelet-derived growth factor-BB after use in extraction socket defects: a case series. Int J Periodontics Restorative Dent 2010; 30: 365-373.

Received: 18 July 2019, Approved: 18 August 2019, Last revised: 20 August 2020 